\title{
Bundling of Power Quality Indices as Method for Correlating Load Susceptibility and Disturbances Superposition
}

\author{
P. Janik ${ }^{1}$, Z. Waclawek ${ }^{1}$, T. Lobos ${ }^{1}$ and H. Amaris ${ }^{2}$ \\ ${ }^{1}$ Department of Electrical Engineering \\ Wroclaw University of Technology \\ Wyb. Wyspianskiego 27, 50-370 Wroclaw, (Poland) \\ Phone:+0048713202901, e-mail: przemyslaw.janik@pwr.wroc.pl \\ ${ }^{2}$ Electric Engineering Department \\ Universidad Carlos III de Madrid, \\ Av. de la Universidad 30, 28911 Leganés (Madrid), (Spain) \\ Phone: +34 916248 777, e-mail: hamaris@ing.uc3m.es
}

\begin{abstract}
This paper presents a neuro-fuzzy approach to power quality assessment. Electrical equipment susceptibility to disturbances varies from type to type. Additionally, not only one physical phenomenon (e.g. harmonics) can cause equipment malfunctioning or damage, but more likely a superposition of some different disturbances. The authors propose an automated neuro-fuzzy approach to power quality indicia bundling which is correlated to equipment susceptibility. Whereby distortion power is seen as one of the power quality indices.

The neuro-fuzzy analyzer matches logically different indices and gives as output one value describing the possible thread to electrical equipment. A reduction of data amount to be analysed by a system engineer is aimed.
\end{abstract}

\section{Key words}

Power Quality, Neuro-Fuzzy System, Disturbances Interference, Reactive Power

\section{Introduction}

Allowed power quality disturbances' levels and acceptable parameters deviations are defined in international standards, e.g. [1], [2]. They are under constant monitoring and supervision. There are numerous reasons for still growing importance of Power Quality (PQ) indices [3],[4], [5]. Nevertheless, it seems advisable to make logical interconnection between equipment malfunction and severity of different disturbances interfering at a time and not only observe power quality indices one by one. In some cases it is not necessary to install sophisticated PQ enhancement devices, because the load does not suffer from a specific disturbance even higher then allowed in standards. On the contrary, superposition and interference of different disturbances which are within limits given in standards may cause malfunction or damage of appliances.
This paper proposes a method for a multi criteria power quality assessment applying a neuro-fuzzy system to handle the dependency between superposition of different disturbing phenomena and specific device susceptibility to disturbances.

Neural networks have the ability do learn and to adapt, so they seem flexible enough to be applied to parameterize an adaptive fuzzy system. Fuzzy logic interference algorithm [6] may be successfully applied to handle the dependence between numerous superimposed disturbances and an unique susceptibility pattern. It was successfully applied for classification in the past [7],[11]. Further, there is a proposal of incorporating the values of reactive power and distortion power as PQ indices into the multi criteria assessment.

The authors aimed to evaluate the applicability of a neuro-fuzzy system for bundling or in other words for joined of disturbing phenomena.

\section{Neuro Fuzzy System}

Fuzzy logic is seen as an extension of binary Boolean logic [8], [9].

In many situations the assumption of crisp membership or non-membership of an element $\mathrm{x}$ to set $\mathrm{A}$ is too restrictive. Contrary to a classical set a fuzzy set is a model in which the transition from membership to nonmembership is gradual rather than abrupt [10]. Such a transition is usually characterized by membership function. A membership function is a curve that defines how each point in the input space (sometimes referred as the universe of discourse) is mapped to a membership value between 0 and 1. Fuzzy sets proposed by Zadeh are uniquely described by their membership functions. A fuzzy set $A$ in the universe of discourse $U$ is defined as a set of ordered pairs:

$$
\mathrm{A}=\left\{\mathrm{x}, \mu_{\mathrm{A}}(\mathrm{x}) \mid \mathrm{x} \in \mathrm{U}\right\}
$$

where $\mu_{A}(x)$ is the membership function of $x$ in $A$. 
Fuzzy logic systems have been developed based on Zadeh's theory. These systems consist of a knowledge base and a reasoning mechanism called fuzzy inference engine [10]. The simple, general structure of a fuzzy system has four blocks (Fig.1) named fuzzification, fuzzy rule base, fuzzy inference engine and defuzzyfication [8]. A fuzzification has the effect of transforming crisp data into fuzzy sets. A fuzzy rule base consists of a collection of fuzzy if-then rules. A fuzzy inference engine combines these rules into a mapping from the input of the system into its output, using fuzzy reasoning methods. The defuzzification block extracts crisp value from the output fuzzy set.

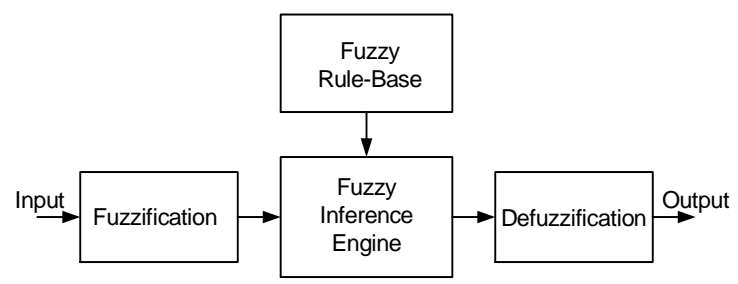

Fig. 1. Fuzzy system structure

The basic problem in fuzzy system design is obtaining a set of fuzzy if-then rules [10]. For automatic fuzzy rules generation an artificial neural network was used as far as there was no expert knowledge to be used instead [11]. In the tests the adaptive neuro-fuzzy inference system (ANFIS) was utilized (Fig. 2), where only measurement information has been processed [12]. The fuzzy rules are implicitly acquired into the network itself. The parameters associated with the membership functions are tuned through the learning process. ANFIS uses a combination of least squares estimation and a backpropagation algorithm for network parameters estimation.

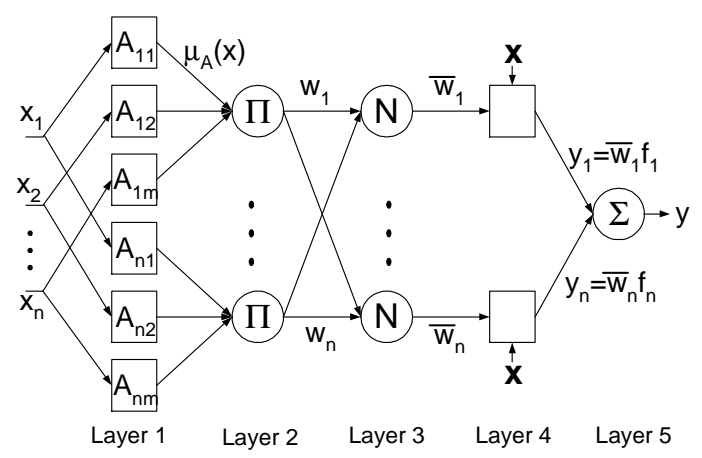

Fig. 2. Adaptive neuro-fuzzy inference system

\section{Power computation for nonsinusoidal currents}

Power factor or the amount of reactive power consumed by the end user is limited. Violation of limits causes financial penalties and technical problems, e.g. distribution line overload [5].

The definition and computation of reactive power in case of nonsinusoidal voltages and currents is a matter of upto-date considerations. Several approaches has been proposed [13], [14].

The authors suggest the application of Budeanu approach to the multi criteria assessment of power quality [13].
A rectangular voltage and corresponding current in a RL branch has been chosen to present proposed method from analytical point of view.

Firstly, the voltage (Fig. 3) may be defined by

$$
u(t)=\left\{\begin{array}{llr}
U_{m} & \text { for } & 0 \leq t \leq 0.5 T \\
0 & \text { for } & 0.5 \mathrm{~T}<\mathrm{t} \leq \mathrm{T}
\end{array}\right.
$$

The voltage, deliberately with DC component and strongly distorted, is feeding a simplified RL branch (Fig.4) where $\mathrm{R}=50 \mathrm{Ohm}, \mathrm{L}=0.2 \mathrm{H}$.

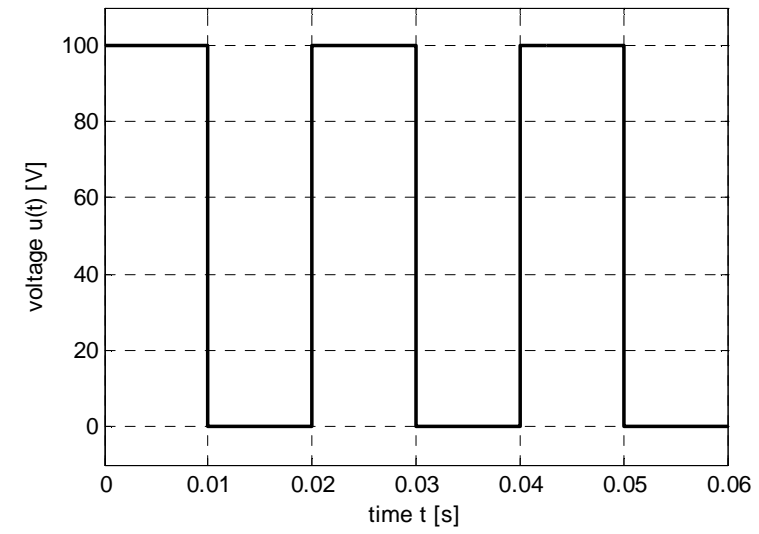

Fig. 3. Rectangular voltage with DC component

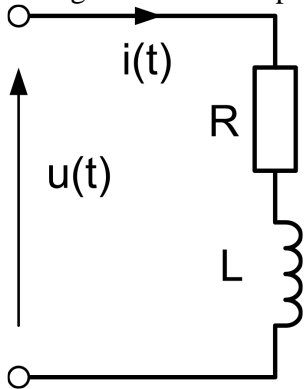

Fig. 4 RL branch fed by a rectangular voltage $u(t)$

Using the expression for the transfer function of the above branch (Fig. 4)

$$
H(s)=\frac{I(s)}{U(s)}=\frac{1}{R+s L}
$$

we can calculate the current

$$
\mathrm{I}(\mathrm{s})=\mathrm{H}(\mathrm{s}) \mathrm{U}(\mathrm{s})
$$

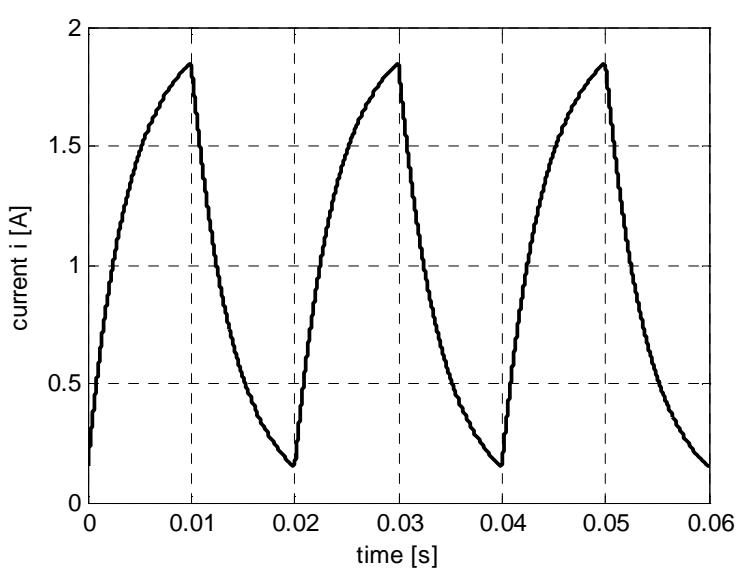

Fig. 5. Current in RL branch by rectangular voltage 
In this particular case the expression for instantaneous current is a result of the computation of inverse Laplace Transform

$$
i(t)=\frac{U_{m}}{R} \begin{cases}1-\frac{\exp \left(-\frac{R}{L} t\right)}{1+\exp \left(-\frac{R T}{2 L}\right)} & \text { for } 0 \leq t \leq 0.5 T \\ \frac{\exp \left(\frac{R T}{2 L}\right) \cdot \exp \left(-\frac{R}{L} t\right)}{1+\exp \left(-\frac{R T}{2 L}\right)} & \text { for } 0.5 T<t \leq T\end{cases}
$$

Having the current (Fig. 5) and voltage (Fig. 3) as analytical functions we can calculate the Fourier components and apply

$$
\mathrm{Q}=\sum \mathrm{U}_{\mathrm{n}} \mathrm{I}_{\mathrm{n}} \sin \varphi_{\mathrm{n}}
$$

For the computation of reactive power and

$$
D=\sqrt{\sum_{n=0}^{N} U_{n}^{2} \sum_{n=0}^{N} I_{n}^{2}-\left(\sum_{n=0}^{N} U_{n} I_{n} \cos \varphi_{n}\right)^{2}+}
$$

For the computation of distortion power. Fig. 6 and Fig. 7 show the amplitudes of harmonic components used for the computation of $\mathrm{Q}$ and $\mathrm{D}$ values.

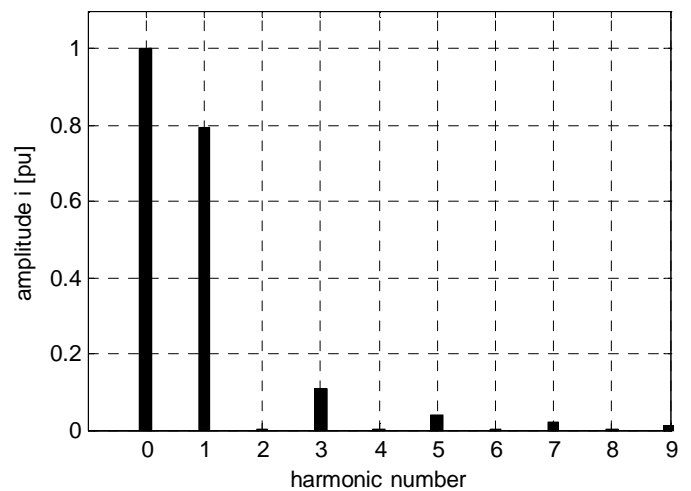

Fig.6 Harmonic content of the current in Fig. 5

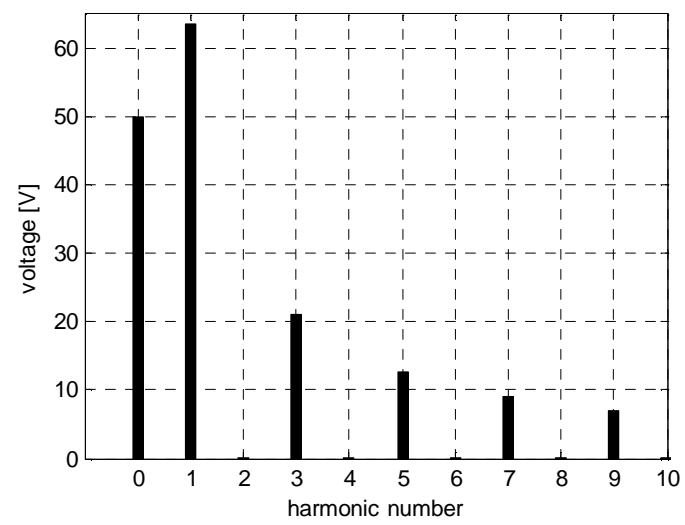

Fig. 7 Harmonic content of the voltage in fig. 3

\section{Simulation Results}

Simulations were carried out to verify the ability of a neural networks to incorporate the knowledge of a artificial test device sensibility to overlaid disturbances. Randomly chosen values of disturbances has been chosen to assess the correctness of recognition in the sense of probability It should be stressed, that it was in the scope of research to find a flexible tool, capable of learning different sensibility patterns. This capability is important for further practical implementations in different environments.

For the preliminary research the sensibility of devices was defined due to arbitral rules. In practice it should be determined in accordance to measurements of disturbances levels and devices malfunctioning rate.

Neural network should distinguish between normal and abnormal condition of supply system (poor power quality). In uncertain cases there should be a proper indication of a "near missed" situation, which could lead to improper operation or damage (probabilistic sense). This property is an important advantage of fuzzy system. Verification of neuro-fuzzy flexibility and adaptability to equipment susceptibility pattern was tested using load pattern susceptible to transients and high order harmonics (e.g. capacitor banks, instrument transformers). The number of input values was intentionally reduced to four: 19. and 21 harmonics (H19, H21), distorted power D, number of overvoltages.

Table I shows names and arbitrary selected change ranges of input parameters, which were simulated for every $10 \mathrm{~min}$. period and correspond (besides distortion power) to standard [1].

Table I -Power quality indices chosen for tests

\begin{tabular}{|c|c|c|c|}
\hline disturbance & \multicolumn{3}{|c|}{ disturbance level } \\
\cline { 2 - 4 } name & allowed & middle & high \\
\hline $\begin{array}{c}\text { 19. harm. } \\
\text { level }\end{array}$ & $0-1.0 \%$ & $1.0-1.5 \%$ & $1.5-2.0 \%$ \\
\hline $\begin{array}{c}\text { 21. harm. } \\
\text { level }\end{array}$ & $0-0.3 \%$ & $0.3-0.5 \%$ & $0.5-0.9 \%$ \\
\hline $\begin{array}{c}\text { distortion } \\
\text { power D }\end{array}$ & $0-10 \%$ & - & $10-20 \%$ \\
\hline overvoltages & $0-9$ & $10-17$ & $18-30$ \\
\hline
\end{tabular}

The equipment susceptibility was predefined and can be arbitrary described as follow: If 19. harmonic or 21 harmonic are high (for details see Table I) abnormal operation will be expected. If two disturbances from three (H19, H21, overvoltages) are middle abnormal operation will be expected. If one disturbance from three (H19, H21, overvoltages) is middle and D is high also abnormal operation will be expected. On the contrary, if $\mathrm{D}$ is high and $\mathrm{H} 19, \mathrm{H} 21$, overvoltages are allowed normal operation will be expected.

The error criterion was the difference between predefined value " 0 " or " 1 " and the network output value. This difference was seen as misclassification for all input values.

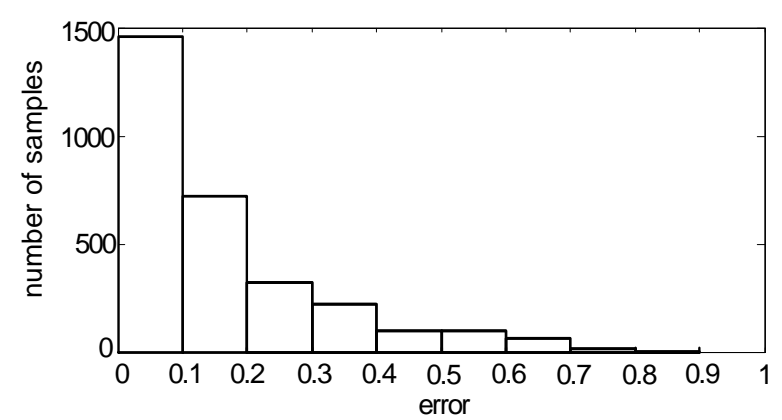

Fig. 8. Erroneous classification histogram for 3000 probes 
The histogram of 3000 runs indicates that almost half of probes was classified without a smallest error. No probe was assigned totally false, with error equal to one.

Selection of detailed results is presented in Table II.

Table II -Results obtained with neuro-fuzzy system

\begin{tabular}{|c|c|c|c|c|c|c|}
\hline$\dot{z}$ & $\frac{\sqrt{9}}{9}$ & $\frac{\bar{s}}{\bar{N}}$ & 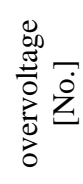 & 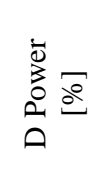 & 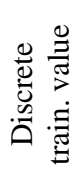 & 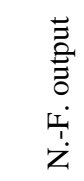 \\
\hline 1. & 1.68 & 0.64 & 25 & 13.58 & 1 & 1.00 \\
\hline 2. & 1.68 & 0.33 & 25 & 18.15 & 1 & 0.99 \\
\hline 3. & 1.41 & 0.76 & 21 & 6.03 & 1 & 0.99 \\
\hline 4. & 0.43 & 0.61 & 18 & 8.13 & 1 & 1.07 \\
\hline 5. & 1.52 & 0.51 & 9 & 7.54 & 1 & 1.04 \\
\hline 6. & 1.97 & 0.09 & 9 & 6.51 & 1 & 0.76 \\
\hline 7. & 0.19 & 0.14 & 20 & 19.52 & 1 & 1.06 \\
\hline 8. & 0.75 & 0.37 & 19 & 11.12 & 1 & 0.95 \\
\hline 9. & 1.82 & 0.72 & 30 & 11.04 & 1 & 0.98 \\
\hline 10. & 0.59 & 0.51 & 15 & 18.71 & 1 & 0.99 \\
\hline 11. & 1.60 & 0.11 & 16 & 3.85 & 1 & 1.00 \\
\hline 12. & 0.23 & 0.57 & 4 & 5.85 & 1 & 0.77 \\
\hline 13. & 1.89 & 0.55 & 24 & 15.89 & 1 & 1.00 \\
\hline 14. & 0.91 & 0.40 & 5 & 13.05 & 1 & 0.64 \\
\hline 15. & 1.68 & 0.11 & 9 & 4.74 & 1 & 0.72 \\
\hline 16. & 0.80 & 0.51 & 13 & 10.74 & 1 & 0.95 \\
\hline 17. & 1.11 & 0.37 & 11 & 9.58 & 1 & 0.73 \\
\hline 18. & 1.63 & 0.27 & 16 & 4.76 & 1 & 1.01 \\
\hline 19. & 1.22 & 0.34 & 23 & 10.19 & 1 & 1.01 \\
\hline 20. & 1.16 & 0.20 & 12 & 7.74 & 1 & 0.63 \\
\hline 21. & 0.20 & 0.30 & 7 & 5.15 & 0 & 0.09 \\
\hline 22. & 0.72 & 0.05 & 3 & 0.70 & 0 & -0.14 \\
\hline 23 & 0.14 & 0.18 & 1 & 4.31 & 0 & -0.16 \\
\hline 24 & 1.13 & 0.16 & 3 & 6.65 & 0 & 0.23 \\
\hline 25 & 0.50 & 0.37 & 3 & 8.77 & 0 & 0.30 \\
\hline 26 & 0.79 & 0.27 & 4 & 6.97 & 0 & 0.05 \\
\hline 27 & 0.85 & 0.31 & 8 & 6.99 & 0 & 0.25 \\
\hline 28 & 0.74 & 0.01 & 9 & 5.87 & 0 & 0.07 \\
\hline 29 & 0.12 & 0.26 & 1 & 8.65 & 0 & -0.04 \\
\hline 30 & 0.26 & 0.28 & 8 & 0.52 & 0 & 0.07 \\
\hline 31 & 0 & 0.29 & 1 & 9.50 & 0 & 0.03 \\
\hline 32 & 1.02 & 0.20 & 8 & 1.71 & 0 & 0.18 \\
\hline 33 & 0.90 & 0.01 & 11 & 3.43 & 0 & 0.27 \\
\hline 34 & 0.44 & 0.35 & 3 & 1.86 & 0 & 0.17 \\
\hline 35 & 0.05 & 0.02 & 12 & 5.62 & 0 & 0.29 \\
\hline 36 & 0.51 & 0.07 & 9 & 3.69 & 0 & 0.03 \\
\hline 37 & 0.85 & 0.30 & 5 & 1.13 & 0 & 0.11 \\
\hline 38 & 0.58 & 0.19 & 4 & 4.43 & 0 & -0.10 \\
\hline 39 & 0.40 & 0.36 & 9 & 7.88 & 0 & 0.37 \\
\hline 40 & 0.62 & 0.24 & 8 & 1.22 & 0 & 0.05 \\
\hline
\end{tabular}

Most cases, acorrdingly to the learning pattern, have been recognized correctly. In some situation there was ambiguity. For example in case $14 \mathrm{D}$ is "high" and $\mathrm{H}_{21}$ is "medium". Overvoltage and $\mathrm{H}_{19}$ are allowed. The system output - 0.64 - may be inerpreted as "danerous" to some degree, but rather not "disasterous".

\section{Conclusion}

Important advantage of presented approach to power quality assessment is the reduction of data to be analyzed by a human system operator. The neuro-fuzzy analyzer matches logically different indices and gives as output one value describing the possible (probable) thread to electrical equipment.

Disadvantageous in the descried procedure is the learning process, for which quite large amount of training vectors is required.

Positive results encourage further research activities. Considered is comparison of the neuro-fuzzy system results with a different classifier (e.g. NN) and utilization of various sets of power quality indicia.

\section{References}

[1] EN-50160 Standard: Voltage characteristics of electricity supplied by public distribution systems

[2] IEC 61000-4-7 Standard: Electromagnetic compatibility (EMC) - Part 4-7: Testing and measurement techniques General guide on harmonics and interharmonics measurements and instrumentation, for power supply systems and equipment connected thereto

[3] M. H. J. Bollen, "Understanding Power Quality Problems. Voltage Sags and Interruptions", IEEE Press, 2000, pp. 1-4.

[4] R. C. Dugan and M. F. McGranaghan and H. W. Beaty, "Electrical Power System Quality", McGraw-Hill, New York, 1996

[5] J. Arrillaga, N. R. Watson, S Chen, "Power System Quality Assessment", Wiley, New York, 2000

[6] W. R. A. Ibrahim and M. M. Morcos, "A Power Quality Perspective to System Operational Diagnosis Using Fuzzy Logic and Adaptive Techniques", IEEE Trans. on Power Delivery, vol 18, No. 3, July 2003, pp.903-909

[7] J. C. Bezdek and S. K. Pal, Fuzzy Models for Pattern Recognition. Methods that Search for Structures in Data, IEEE Press, New York, 1992

[8] C. T. Leondes, "Fuzzy Theory Systems. Techniques and Applications", Academic Press, New York, 1999

[9] C.H. Chen, "Fuzzy Logic and Neural Network Handbook", McGraw-Hill, New York, 1996

[10] E. Czogała. and J. Łeski., "Fuzzy and Neuro-Fuzzy Inteligent Systems", Physica-Verlag, Heidelberg, 2000

[11] R. K. Aggarwal, M. Joorabian, Y. H. Song, "Fuzzy neural network approach to accurate transmission line fault location", Engineering Intelligent Systems, Vol 5, No. 4, 1997

[12] J.W. Hines, MATLAB Supplement to Fuzzy and Neural Approaches in Engineering, John Wiley \& Sons, New York, 1997

[13] L. S. Czarnecki "Budeanu and Fryze: Two frameworks for interpreting power properties of circuits with nonsinusoidal voltages and currents", Electrical Engineering 80 (I997), pp.359-367

[14] A.E. Emanuel, "Powers in nonsinusoidal situations. A review of definitions and phisical meaning.", IEEE Transactions on Power Delivery, vol.5, No.3, pp. 1377-1389 